\title{
Towards Non-Invasive Brain-Computer Interface for Hand/Arm Control in Users With Spinal Cord Injury
}

\author{
Gernot R. Müller-Putz, Joana Pereira, Patrick Ofner, Andreas Schwarz, Catarina Lopes Dias, \\ Reinmar J. Kobler, Lea Hehenberger, Andreas Pinegger, Andreea I. Sburlea
}

Institute of Neural Engineering

Graz University of Technology

Graz, Austria

E-mail: gernot.mueller@tugraz.at

\begin{abstract}
Spinal cord injury (SCI) can disrupt the communication pathways between the brain and the rest of the body, restricting the ability to perform volitional movements. Neuroprostheses or robotic arms can enable individuals with SCI to move independently, improving their quality of life. The control of restorative or assistive devices is facilitated by braincomputer interfaces (BCIs), which convert brain activity into control commands.

In this paper, we summarize the recent findings of our research towards the main aim to provide reliable and intuitive control. We propose a framework that encompasses the detection of goaldirected movement intention, movement classification and decoding, error-related potentials detection and delivery of kinesthetic feedback. Finally, we discuss future directions that could be promising to translate the proposed framework to individuals with SCI.
\end{abstract}

Keywords- brain-computer interface, EEG, spinal cord injury, movement decoding, intuitive control

\section{INTRODUCTION}

Many everyday goal-directed interactions, such as grasping a cup of coffee, are not possible for people who received a cervical spinal cord injury (SCI). The communication pathways between the brain and the peripheral nervous system can be affected, depending on the level and severity of the damage to the spinal cord. To improve the quality of life of individuals with SCI, and help them regain independence in movement, we aim to record their electroencephalographic (EEG) brain signals and transform these signals into continuous control commands for a restorative neuroprosthesis or robotic arm.

We have shown that such control signals can be obtained from a brain-computer interface (BCI) and used for motor control [1]. For instance, one approach to switch between different grasp phases is the sustained motor imagery (MI) of hand or feet movements [2], [3]. Furthermore, we have shown that imagined movements with different durations could be used, both in healthy and in end-users with SCI, for motor control [4]-[6].

The advantages of BCI for motor control can be augmented by adding other input modalities (e.g. shoulder position sensor) leading to a complementary system, the hybrid BCI [7]-[9]. Although such systems have shown promising results [10], [11], they still lack an intuitive control. This drawback could be overcome if the movements decoded by the system can closely reflect the user's intention [12].

In the next section, we will present some concepts of our framework: goal-directed movement intention detection, classification of single upper limb movements and grasp types, kinematics decoding and error-related potentials detection.

\section{METHODS \& RESUlTS}

\section{A. Goal-directed movement intention}

We are currently studying the cognitive processes which allow a decision on motor goals and lead to the initiation of movement. In [13], we investigated whether the detection of movement intention was influenced by the goal-directedness of the movement in a movement execution (ME) task. We used low-frequency time-domain EEG features in a single-trial classification scheme and found that the presence of a motor goal had a positive influence on the classifier performance, when compared to a non-goal-directed condition. More specifically, the highest classification accuracies were achieved when the goal-directed movement condition was executed (grand-average of 79\%). Notably, before actual movement onset, results were above chance level for all subjects for the goal-directed movement condition, while only 6 out of 10 subjects were above chance level for the non-goaldirected condition.

In another study [14], we designed a paradigm to separate target selection from the actual motor task, which allowed us to study the differences between externally and internallydriven target selection. Differences between conditions were observed in the positive late component of the event-related potential (ERP). Further, this paradigm allowed us to have an estimation of the onset of a self-paced MI task and study the movement-related cortical potentials (MRCPs) to assess suitability of MI for the detection of movement intention. 


\section{B. Classification of single upper limb movements}

Discrimination of several single and non-repetitive upper limb movements is also possible using the time-domain of low-frequency EEG [15]. Fifteen healthy subjects executed and imagined six different sustained upper limb movements. We applied a multi-class classification of 6 movements: elbow flexion/extension, forearm supination/pronation, and hand open/close. Additionally, these movements were classified against a rest class. We obtained significant average classification accuracies of $55 \%$ (movement vs. movement) and $87 \%$ (movement vs. rest) for $\mathrm{ME}$, and $27 \%$ and $73 \%$, respectively, for MI. By analyzing the classifier patterns in the source space, we could show that for ME, mainly motor areas $\mathrm{M} 1, \mathrm{~S} 1$, premotor and posterior parietal cortex contained the discriminable movement-related information.

\section{Classification of grasp types}

Despite the high number of degrees of freedom of the human hand, most actions of daily life can be executed incorporating only palmar, pincer and lateral grasp. We showed that these three different executed reach-and-grasp actions are discriminable using EEG [16]. Using lowfrequency time-domain features, we achieved binary classification accuracies of $72.4 \%$ (grasp vs. grasp) and $93.5 \%$ (grasp vs. rest). In an offline multi-class classification scenario which incorporated not only all reach-and-grasp actions but also the rest condition, classification performance peaked at $65.9 \%$. These findings will eventually contribute to our attempt of controlling a grasp neuroprosthesis.

\section{Classification of movements in end-users}

Transferring the results to a study with five end-users with high SCI (Neurological level: C3 - C5), we investigated 2 smaller subsets, i.e. types of movements, of the two aforementioned studies [15], [16]. For the single upper limb movements (subset 1), a classification accuracy of $53 \%$ was achieved. Grasps (subset 2) could also be classified from MRCP features with an average accuracy of 57\% [17].

\section{E. Kinematics decoding and EOG artifacts}

Complementary to classifying hand/arm movements, we have also investigated decoding of movement kinematics. In [18], 3D hand velocities (and positions) significantly correlated with their neural estimates $(r=0.7$ across dimensions) for a continuous self-paced arm movement task in a healthy population. However, transferring this approach to the end-users is not trivial, since their condition impedes arm/hand movements, and consequently prevents recording of the ground truth kinematic signals. We showed in a healthy population that it is feasible to circumvent this limitation by switehing to an imagery task and designing the paradigm so that the ground truth can be reliably estimated. In [19], we used a metronome as an auditory pacemaker to induce rhythmic arm movement imaginations.

Another option is to study overt orienting in visuomotor (VM) tasks. These tasks involve visual stimuli and feedback; e.g. a target stimulus' position in space or distance between a target and an end-effector. Intuitively, subjects would orient attention to objects of interest and/or track them by moving their eyes. If the experimental protocol does not impose the additional task of inhibiting these eye movements, electrooculogram (EOG) artifacts arise.

An ocular artifact correction approach is required to (1) be suitable for online experiments, (2) attenuate the artifacts to noise level, and (3) assure invariance to cortical sources, considering the fact that eye movements and brain activity can be substantially correlated in VM tasks. In [20], we addressed constraints (1) and (2) by applying a block design based experimental approach. In the first block, we recorded calibration data. The data was intended to fit model parameters of 5 EOG artifact correction algorithms. We then evaluated the algorithms' correction quality and their influence on resting EEG data of a second block, recorded 1 hour later. We found that an algorithm, based on artifact subspace subtraction [21], could attenuate horizontal and vertical eye movement artifacts to chance level, and maintain resting brain activity (root mean squared error between resting EEG before and after correction $<1.5 \mu \mathrm{V})$.

\section{F. Error-related Potentials}

The incorporation of error-related potentials (ErrPs) detection into a BCI can improve its performance, contributing to a swifter interaction with the users.

We recorded 8 subjects during a task with continuous control, in which, in some trials, an erroneous event happened (error trials). The feedback was continuous and either unmasked (normal feedback) or masked [22]. The masked feedback intended to mimic the instability resulting from decoding the cursor's position from brain signals and was obtained by adding a variable noise component perpendicular to the velocity vector of the cursor. The time-locked classification of masked error trials against unmasked error trials resulted in, on average, $59.1 \%$ of the masked error trials being successfully classified and $59.5 \%$ of the unmasked error trials being successfully classified. The time-locked classification of correct trials against error trials (when considering both masked and unmasked trials together) resulted in, on average, $94.3 \%$ of the correct trials being successfully classified and $80.3 \%$ of the error trials being successfully classified.

\section{DISCUSSION}

In our framework, we envision an end-user with spinal cord injury sitting in a wheelchair. He would like to grasp the cup of coffee located on the table in front of him, by sending his motor command to a robotic arm. Once the robot starts the movement, it follows a path derived from EEG signals towards the target. The robot could also misinterpret the motor commands and reach the wrong target, leading to an error. During the reaching and grasping movements, we would deliver continuous kinaesthetic feedback to a sensory receptive area of the body (e.g. the shoulder).

With respect to the movement intention detection, our findings suggest that the detection of movement intention is increased when the movement has a specific goal [13]. Moreover, we also found out that several movements of the 
upper limb have distinct brain patterns and that these differences can be exploited for BCI control [15], [16]. Our first offline results in end-users with SCI seem promising [17], however, it is now mandatory to test the detectors and classifiers online and asynchronously.

Any movement can be achieved by differently combining muscle contractions and joint positions. An interesting question is: How does the brain decide which combination is suitable for a particular movement? One theory is that the brain simplifies this problem by encoding particularly useful patterns of joint movements as distinct units or "synergies". A given task can then be performed by selecting from a small number of synergies, avoiding the need to choose between huge numbers of options every time a movement is intended [23]. Therefore, we want to investigate, in a wider range of movements, the relation between their neural and behavioral activity patterns at a synergetic level.

If an end-user is actually in control of the robotic arm, feedback allows the brain to estimate the arm's state over time and adjust its trajectory. The absence of genuine kinaesthetic feedback from the robotic arm requires the user to closely monitor any movement and its interactions with objects visually. Accurate vision-based state estimation naturally leads to eye movements and in turn to EOG artifacts. Our findings suggest that these artifacts can be selectively attenuated to noise level in an online fashion with little disturbance to the resting EEG. Further evaluation with regard to their influence on trajectory decoding and established movement-related phenomena such as the MRCP and event-related desynchronization/synchronization (ERD/ERS) is required.

Relying on visual feedback alone places a large load on the visual system and potentially affects the performance of complex regulatory processes such as grasping. Moreover, it leads to the control feeling artificial, rather than a genuine extension of one's own body. To facilitate intuitive control, it is imperative to provide supplementary feedback via somatosensory channels, such as proprioceptive or haptic feedback.

The delivery of meaningful somatosensory feedback encompasses proper adjustment of a multitude of parameters and degrees of freedom, such as choosing the feedback modality (e.g. vibrotactile, pressure or electrotactile), determining suitable stimulation loci, or aptly encoding the information, to name just a few. Moreover, taking into account e.g. inter-subject variations of sensitivity profiles (in general, and among individuals with SCI in particular) requires a certain degree of adaptability. We are pursuing investigations into vibrotactile feedback, its potential to enhance intuitive prosthesis control and its effects on EEG movement-related features.

When the BCI misinterprets the brain signals from the enduser, it initiates an unintended command. For the user, the mismatch between the expected action and the received feedback leads to the generation of an ErrP. In our study [22], masked and unmasked ErrPs were indistinguishable in terms of time-locked classification. This indicates that the potential instability resulting from decoding the trajectory of the robotic arm directly from brain signals, will not hinder the decoding of ErrPs in an online scenario. In order to detect and correct such mismatches during the continuous movement of the robotic arm, we would need an asynchronous detector of errors. In the future, we will investigate the asynchronous decoding of ErrPs during a task with continuous control and continuous feedback.

\section{CONCLUSION}

In conclusion, we evaluated our framework in a large number of healthy users, as well as in some end-users with high SCI, and we obtained promising results Future studies are needed to confirm if imagined or attempted movements can be classified/ decoded online from individuals with SCI and if the BCI performance is sufficient to reliably control a neuroprosthesis or a robotic arm. It also has to be determined if the BCI performances can be maintained and/or increased by user training. Furthermore, the translation of the ErrPs detection and kinaesthetic feedback delivery needs to be assessed in end-users with SCI.

\section{ACKNOWLEDGMENTS}

This work was supported by the EU ICT Programme Project H2020-643955 MoreGrasp, and the ERC Consolidator Grant ERC-681231, Feel Your Reach.

\section{REFERENCES}

[1] G. R. Müller-Putz, R. Scherer, G. Pfurtscheller, and R. Rupp, "Braincomputer interfaces for control of neuroprostheses: from synchronous to asynchronous mode of operation / Brain-Computer Interfaces zur Steuerung von Neuroprothesen: von der synchronen zur asynchronen Funktionsweise," Biomedizinische Technik/Biomedical Engineering, vol. 51, no. 2, pp. 57-63, Jul. 2006.

[2] G. Pfurtscheller, G. R. Müller, J. Pfurtscheller, H. J. Gerner, and R. Rupp, "'Thought' - control of functional electrical stimulation to restore hand grasp in a patient with tetraplegia," Neurosci. Lett., vol. 351, no. 1, pp. 33-36, Nov. 2003.

[3] G. R. Müller-Putz, R. Scherer, G. Pfurtscheller, and R. Rupp, "EEGbased neuroprosthesis control: a step towards clinical practice," Neurosci. Lett., vol. 382, no. 1-2, pp. 169-174, Apr. 2005.

[4] G. R. Müller-Putz, R. Scherer, G. Pfurtscheller, and C. Neuper, "Temporal coding of brain patterns for direct limb control in humans," Front. Neurosci., 2010.

[5] Z. Wu et al., "The Convergence of Machine and Biological Intelligence," IEEE Intell. Syst., vol. 28, no. 5, pp. 28-43, 2013.

[6] R. Rupp et al., "Think2grasp - BCI-Controlled Neuroprosthesis for the Upper Extremity," Biomed. Tech. , Sep. 2013.

[7] G. Pfurtscheller et al., "The hybrid BCI," Front. Neurosci., 2010.

[8] G. R. Müller-Putz et al., "Tools for brain-computer interaction: a general concept for a hybrid BCI," Front. Neuroinform., vol. 5, 2011.

[9] M. Rohm et al., "Hybrid brain-computer interfaces and hybrid neuroprostheses for restoration of upper limb functions in individuals with high-level spinal cord injury," Artif. Intell. Med., vol. 59, no. 2, pp. 133-142, Oct. 2013.

[10] G. R. Müller-Putz et al., "Towards Noninvasive Hybrid Brain-Computer Interfaces: Framework, Practice, Clinical Application, and Beyond," Proc. IEEE, vol. 103, no. 6, pp. 926-943, 2015.

[11] R. Rupp, M. Rohm, M. Schneiders, A. Kreilinger, and G. R. MullerPutz, "Functional Rehabilitation of the Paralyzed Upper Extremity After Spinal Cord Injury by Noninvasive Hybrid Neuroprostheses," Proc. IEEE, vol. 103, no. 6, pp. 954-968, 2015. 
[12] G. R. Müller-Putz, A. Schwarz, J. Pereira, and P. Ofner, "From classic motor imagery to complex movement intention decoding," in Progress in Brain Research, 2016, pp. 39-70.

[13] J. Pereira, P. Ofner, A. Schwarz, A. I. Sburlea, and G. R. Müller-Putz, "EEG neural correlates of goal-directed movement intention," Neuroimage, vol. 149, pp. 129-140, 2017.

[14] J. Pereira, A. I. Sburlea, and G. R. Müller-Putz, "Event-Related Potentials in Externally and Internally-Driven Target Selection: a preliminary study," in Proceedings of the 7th Graz Brain-Computer Interface Conference 2017 From Vision to Reality, Graz, Austria, 2017, pp. 400-405.

[15] P. Ofner, A. Schwarz, J. Pereira, and G. R. Müller-Putz, "Upper limb movements can be decoded from the time-domain of low-frequency EEG," PLoS One, vol. 12, no. 8, p. e0182578, Aug. 2017.

[16] A. Schwarz, P. Ofner, J. Pereira, A. I. Sburlea, and G. R. Müller-Putz, "Decoding natural reach-and-grasp actions from human EEG," J. Neural Eng., 2017.

[17] G. R. Müller-Putz, A. Schwarz, J. Pereira, P. Ofner, A. Pinegger, and R. Rupp, "EEG-controlled grasp neuroprosthesis for individuals with high spinal cord injury - decoding of multiple single limb movements," in 21 st Annual Conference of the International Functional Electrical Stimulation Society, London, UK.
[18] P. Ofner and G. R. Müller-Putz, "Decoding of velocities and positions of 3D arm movement from EEG," Conf. Proc. IEEE Eng. Med. Biol. Soc., vol. 2012, pp. 6406-6409, 2012.

[19] P. Ofner and G. R. Müller-Putz, "Using a Noninvasive Decoding Method to Classify Rhythmic Movement Imaginations of the Arm in Two Planes," IEEE Transactions on Biomedical Engineering, vol. 62, no. 3, pp. 972-981, 2015.

[20] R. J. Kobler, A. I. Sburlea, and G. R. Müller-Putz, "A Comparison of Ocular Artifact Removal Methods for Block Design Based Electroencephalography Experiments," in Proceedings of the 7th Graz Brain-Computer Interface Conference 2017, Graz, 2017, pp. 236-241.

[21] L. C. Parra, C. D. Spence, A. D. Gerson, and P. Sajda, "Recipes for the linear analysis of EEG," Neuroimage, vol. 28, no. 2, pp 326-341, 2005.

[22] C. Lopes Dias, A. I. Sburlea, and G. R. Müller-Putz, "Error-related Potentials with Masked and Unmasked Onset during Continuous Control and Feedback," in Proceedings of the 7th Graz Brain-Computer Interface Conference 2017, Graz, 2017, pp. 279-284.

[23] H. H. Ehrsson, J. P. Kuhtz-Buschbeck, and H. Forssberg, "Brain regions controlling nonsynergistic versus synergistic movement of the digits: a functional magnetic resonance imaging study," J. Neurosci., vol. 22, no. 12, pp. 5074-5080, Jun. 2002. 